\title{
Weak Measurements with Orbital-Angular-Momentum Pointer States
}

\author{
G. Puentes, ${ }^{1, *}$ N. Hermosa, ${ }^{1}$ and J. P. Torres ${ }^{1,2}$ \\ ${ }^{1}$ ICFO-Institut de Ciencies Fotoniques, Mediterranean Technology Park, 08860 Castelldefels, Barcelona, Spain \\ ${ }^{2}$ Department Signal Theory and Communications, Campus Nord D3, Universitat Politecnica de Catalunya, 08034 Barcelona, Spain
}

(Received 16 April 2012; published 24 July 2012)

\begin{abstract}
Weak measurements are a unique tool for accessing information about weakly interacting quantum systems with minimal back action. Joint weak measurements of single-particle operators with pointer states characterized by a two-dimensional Gaussian distribution can provide, in turn, key information about quantum correlations that can be relevant for quantum information applications. Here we demonstrate that by employing two-dimensional pointer states endowed with orbital angular momentum $(\mathrm{OAM})$, it is possible to extract weak values of the higher order moments of single-particle operators, an inaccessible quantity with Gaussian pointer states only. We provide a specific example that illustrates the advantages of our method both in terms of signal enhancement and information retrieval.
\end{abstract}

DOI: 10.1103/PhysRevLett.109.040401

PACS numbers: 42.50.Tx, 03.65.Ta, 42.50.Xa

Ideal von Neumann measurements in quantum mechanics are able to distinguish between the different eigenstates of a given observable. Contrary to this, weak measurements, a concept first introduced by Aharonov, Albert, and Vaidman [1], describe a situation where the coupling between the measuring device and the observable is a weak perturbation. In this case, the uncertainty in the measurement is large in comparison with the separation between the eigenvalues of the observable, such that the different outcomes cannot be resolved.

What makes a weak measurement an interesting phenomenon is that the weak value of an observable $A$ of the system can yield an unexpected result [2-4]. In particular, given a preselected state $|i\rangle$ and a postselected state $|f\rangle$ of the system, the weak value $\langle A\rangle_{w}$ is defined as

$$
\langle A\rangle_{w}=\frac{\langle f|A| i\rangle}{\langle f \mid i\rangle} .
$$

If an appropriate postselection is made, for instance by choosing $|i\rangle$ and $|f\rangle$ to be nearly orthogonal, the weak value $\langle A\rangle_{w}$ can be significantly outside the spectrum of $A$ (weak-value amplification), and it can even take imaginary values.

In the case of an observable $A$, which is weakly coupled to a one-dimensional pointer state, the Hamiltonian of the weakly coupled system can be described by $H=g A P_{x}$, where $P_{x}$ is the pointer momentum operator, conjugate to its position operator $X$. For a sufficiently weak coupling strength $g t$, the interaction shifts the mean position of the pointer by an amount $\Delta x=g t \operatorname{Re}\left(\langle A\rangle_{w}\right)$, where $t$ is the duration of the interaction. By measuring the mean value of the pointer position displacement $\langle X\rangle$ and the pointer momentum displacement $\left\langle P_{x}\right\rangle$, it is possible to obtain either the real part of the weak value $\operatorname{Re}\left(\langle A\rangle_{w}\right)$ or its imaginary part $\operatorname{Im}\left(\langle A\rangle_{w}\right)[5]$, thus providing a full measurement of the weak value of the observable $\langle A\rangle_{w}$.
Several successful experimental implementations of weak measurements have been accomplished up to date in a wide range of scenarios: for demonstrating waveparticle duality in the context of cavity-QED [6,7]; for characterizing the response function of a highly dispersive system [8]; for the realization of Leggett-Garg inequality violations [9]; for detecting tiny spatial shifts [10,11] and tiny temporal shifts [12], or tiny beam deflections [13], of intense optical beams; for the direct measurement of the wave function of a quantum system [14], or the weak value of the polarization degrees of freedom of entangled photon pairs [15].

In many applications, it can be important to access the mean value of products of single-particle operators, i.e., $\langle A B\rangle$. Such joint measurements are of great relevance for quantum information since they can contain information about quantum correlations (entanglement) between different degrees of freedom, as for instance in cluster-state quantum computation [16]. However, a strong measurement of joint mean values requires a nonlinear Hamiltonian, which in many cases can prove hard to engineer [17]. Resch and Steinberg [5] circumvented this limitation by employing a two-dimensional Gaussian pointer state and a weakly coupling linear Hamiltonian of the form $H=g_{A} A P_{x}+g_{B} B P_{y}$, where $\left(P_{x}, P_{y}\right)$ are the pointer momentum operators, conjugate to the pointer position operators $(X, Y)$. By performing a second order expansion in the two-dimensional pointer displacement $\langle X Y\rangle$, the authors showed that it is possible to extract the real part of the joint weak value $\langle A B\rangle_{w}$, for the case of commuting observables $[A, B]=0$.

In this Letter, we show that by employing pointer states endowed with orbital angular momentum (OAM), it is possible to retrieve a wider range of second order weak values. An important limitation of Gaussian pointer states is that, due to symmetry properties, they can not provide access to weak values of higher order moments of singleparticle operators, such as $\left\langle A^{n}\right\rangle_{w}$ or $\left\langle B^{n}\right\rangle_{w}$, or to higher 
order moments of joint operators, such as $\left\langle A^{n} B\right\rangle_{w}$ or $\left\langle B^{n} A\right\rangle_{w}(n>1)$, for the case $[A, B]=0$ [5]. Pointer states with OAM introduce a different symmetry for the expectation values of operators and thus provide access to the weak values of higher order moments of single-particle operators and joint operators, a result which is not attainable with Gaussian pointer states.

Moreover, in many applications employing Gaussian pointer states, the weak amplification factor can only be attained in the imaginary component of the weak value. This can be seen as an advantage, as the amplified imaginary part can have a simpler operational interpretation than its real counterpart, since it is not tied to the conditioned average value of an observable, and is rather linked to the measurement back action [5,18]. However, in order to extract such imaginary weak values, additional measurements on the pointer's conjugate variable are required. Here we will show that OAM pointer states can provide access to the enhanced imaginary part of higher order weak values, thus outperforming Gaussian pointer states in terms of signal enhancement and information retrieval.

Finally, we note that since the analytical determination of upper bounds on amplification under the assumption of Gaussian pointer wave function [19], much effort has been devoted to the engineering of optimal probe states [20]. Here, we present a realistic, and experimentally feasible, pointer distribution that can provide significant advantages over Gaussian probe states and that could find several applications both in the context of quantum information and foundational tests of quantum mechanics [21], as well as in weak measurements of cosmological effects, such as in gravitational wave detection with higher order Laguerre-Gauss beams [22], or the back action of the Hawking radiation from a black hole [23].

Consider the weak interaction between two observables $A$ and $B$ of a single-particle system initially prepared in the state $|i\rangle$, with a measuring device initially prepared in the state $|\phi\rangle=\int d x d y \phi(x, y)|x, y\rangle$. The total input state is $\left|\psi^{\mathrm{in}}\right\rangle=|i\rangle \otimes|\phi\rangle$. The Hamiltonian of weak interaction $H=g_{A} A P_{x}+g_{B} B P_{y}$ describes the coupling of observable $A$ with the $x$ dimension of the measuring device while observable $B$ is coupled to its $y$ dimension. Here $x$ and $y$ are cartesian coordinates, and their associated position and momentum quantum operators satisfy the usual commutation rules $[X, Y]=0$ and $\left[P_{x}, P_{y}\right]=0$, respectively. Also, we restrict to the case of commuting observables of the form $[A, B]=0$, though more complex expressions can also be obtained in the case where $[A, B] \neq 0$.

We are interested in the mean value of the operator $O_{X Y}=|f\rangle\langle f| X Y$ at time $t$, where the projector $|f\rangle\langle f|$ performs the postselection operation on the system, and commutes with all the spatial observables for the measuring device. The time-dependent mean value $\left\langle O_{X Y}(t)\right\rangle$ can be obtained by expanding the Heisenberg's equation of motion to second order in the coupling parameters $g_{A}$ and $g_{B}[5]$

$$
\begin{aligned}
\left\langle O_{X Y}(t)\right\rangle= & \left\langle O_{X Y}(0)\right\rangle+\frac{i t}{\hbar}\left\langle\left[H, O_{X Y}\right]\right\rangle \\
& -\frac{t^{2}}{2 \hbar^{2}}\left\langle\left[H,\left[H, O_{X Y}\right]\right]\right\rangle .
\end{aligned}
$$

The two-dimensional pointer states considered here are described by Laguerre-Gauss (LG) modes. LG modes are a set of solutions of the paraxial wave-equation [24], characterized by two integer indices $p$ and $l$. The index $p$ is a positive integer, and $p+1$ determines the number of zeroes of the field along the radial direction. The winding index $l$, which can take any integer number, determines the azimuthal phase dependence of the mode, which is of the form $\sim \exp (i l \varphi)$. Each mode carries a well-defined orbital angular momentum of $l \hbar$ per photon, associated with their spiral wave fronts [25]. The OAM states of light allow for a relatively simple experimental generation, filtering, detection, and control [26]. In this paper, we will concentrate on the case $p=0$ and $l=0, \pm 1$ since this is enough to demonstrate the benefits of using OAM pointer states.

Specifically, the two-dimensional pointer distribution considered here is described by [27]

$$
\phi(x, y)=N[x+i \operatorname{sgn}(l) y]^{|l|} \exp \left(-\frac{x^{2}+y^{2}}{4 \sigma^{2}}\right),
$$

where $\sigma$ is the uncertainty in the pointer state, and $N$ is a normalizing constant so that $\int d x d y|\phi(x, y)|^{2}=1$. The case $l=0$ corresponds to a pointer state with a 2D-Gaussian distribution. In this case, the pointer state is factorable in the two directions, and therefore can not be used to retrieve higher order weak moments of $A$ and $B$. The case $l= \pm 1$ corresponds to states endowed with orbital angular momentum (OAM). Now the pointer distribution is no longer factorable, and this is a key factor to retrieve higher order weak moments of the form $\left\langle A^{2}\right\rangle_{w}$ and $\left\langle B^{2}\right\rangle_{w}$, as it will be shown below. Moreover, we note that by considering larger values of $l$, it should be possible to access weak values of a wider range of moments of single-particle operators and joint operators.

Inspection of Eq. (2), and symmetry properties of Gaussian integrals related to the specific shape of the pointer states given by Eq. (3), show that the first non-zero term is $\left\langle\left[H,\left[H, O_{X Y}\right]\right]\right\rangle$, which is second order in the coupling parameters. Higher order terms do not vanish either, but they can be considered negligible if the coupling constants $g_{A}$ and $g_{B}$, and the duration of the interaction $t$, are sufficiently small with respect to the pointer uncertainty $\sigma$. By making use of $\left\langle\phi\left|P_{x} X\right| \phi\right\rangle=\left\langle\phi\left|P_{y} Y\right| \phi\right\rangle=-i \hbar / 2$, and $\left\langle\phi\left|X P_{x}\right| \phi\right\rangle=\left\langle\phi\left|Y P_{y}\right| \phi\right\rangle=i \hbar / 2$, and due to symmetry properties of the chosen pointer states, we obtain

$$
\begin{aligned}
\langle X Y\rangle= & \frac{g_{A} g_{B} t^{2}}{2}\left[\operatorname{Re}\left(\langle A B\rangle_{w}\right)+\operatorname{Re}\left(\langle A\rangle_{w}^{*}\langle B\rangle_{w}\right)\right] \\
& +l \frac{t^{2}}{2}\left[g_{A}^{2} \operatorname{Im}\left(\left\langle A^{2}\right\rangle_{w}\right)+g_{B}^{2} \operatorname{Im}\left(\left\langle B^{2}\right\rangle_{w}\right)\right] .
\end{aligned}
$$


For $l=0$ (Gaussian pointer states), we recover the result given in Ref. [5]. For $l= \pm 1$ (OAM pointer states), we obtain additional terms proportional to $g_{A}^{2} t^{2} \operatorname{Im}\left(\left\langle A^{2}\right\rangle_{w}\right)$ and $g_{B}^{2} t^{2} \operatorname{Im}\left(\left\langle B^{2}\right\rangle_{w}\right)$, thus providing access to the imaginary part of the second order weak values, a quantity which cannot be retrieved using Gaussian pointer states only.

Note that in an experiment one measures $\langle X Y\rangle$, which contains both the joint weak value $\langle A B\rangle_{w}$ and the squared weak values of single-particle operators $\left\langle A^{2}\right\rangle_{w}$ and $\left\langle B^{2}\right\rangle_{w}$. In order to address the second order weak moments of $A$ and $B$, we can perform two separate measurements: a first one with a Gaussian pointer state, thus obtaining Eq. (4) with $l=0$, and a second one with an OAM pointer state. By subtracting these two measurements, it is possible to obtain the imaginary part of the second order weak value. Also, by measuring the combined position-momentum pointer shift $\left\langle Y P_{x}\right\rangle$, it is possible to access a term proportional to $\operatorname{Re}\left(\left\langle A^{2}\right\rangle_{w}\right)$ while by measuring the complementary shift $\left\langle X P_{y}\right\rangle$, one can access a term proportional to $\operatorname{Re}\left(\left\langle B^{2}\right\rangle_{w}\right)$. In this way it is possible to extract the real parts of the second order weak values. Second order weak values for operators $A$ or $B$ can also be addressed separately, by considering independent Hamiltonians (i.e., taking either $B \equiv 0$, or $A \equiv 0$, respectively), as explained below.

We now consider in detail the case of a single observable weakly coupled to the measuring device (i.e., $B \equiv 0$ ). Equation (4) shows that for $|l|>0$, an interaction that only couples the observable $A$ to the pointer can show a nonzero expectation value for $\langle X Y\rangle$ while for Gaussian pointer states $(l=0)$ this is not the case. Specifically, for pointer states endowed with OAM, we obtain

$$
\langle X Y\rangle=l \frac{\left(g_{A} t\right)^{2}}{2} \operatorname{Im}\left(\left\langle A^{2}\right\rangle_{w}\right) .
$$

Equation (5) shows that it is possible to measure a value for $\langle X Y\rangle \neq 0$, even when the weak interaction does not couple the $y$ dimension of the pointer with the observable $A$ directly. This is due to the fact that the pointer state given in Eq. (3) is not factorable in the $x$ and $y$ dimensions so the coupling of the observable $A$ with the $x$ dimension of the pointer also affects its $y$ dimension. Equation (5) is quite significant as it readily shows that by employing OAM pointer states, and by measuring the corresponding twodimensional pointer shift $\langle X Y\rangle$, we can obtain an imaginary weak value. We note that the imaginary part of the weak value provides information about the instantaneous rate of change in the probability due to the measurement process (i.e., back action mechanism) $[5,18]$. Such rate of change is usually linked to the pointer's conjugate variable. In our case, the nonfactorability of the two-dimensional pointer spatial distribution produces a similar effect in the spatial domain, which can, in turn, be exploited to amplify the spatial shift in a situation where Gaussian states cannot provide an equivalent enhancement factor.

Finally, we present a simple example that, nevertheless, fully demonstrates the advantages of employing OAM pointer states both in terms of signal amplification and information retrieval. We consider a specific configuration that can be experimentally implemented. This makes use of the ideas described above and will help to clearly reveal the advantages of employing an OAM pointer state versus its Gaussian counterpart. Consider a specific observable given by a spin- $1 / 2$ matrix $A$ or the polarization degrees of freedom of the radiation field. Such observable, which can be regarded generally as a linear combination of Pauli matrices [28], has the following form

$$
A=\left(\begin{array}{cc}
9 / 5 & 2 i / 5 \\
-2 i / 5 & 6 / 5
\end{array}\right)
$$

To see its physical meaning in a weak measurement context, we can calculate its eigenstates $a$ and $b$ and the corresponding eigenvalues $\lambda_{1}$ and $\lambda_{2}$, respectively

$$
\begin{aligned}
& \lambda_{1}=1, \quad|a\rangle=(1 / \sqrt{5})(|H\rangle+2 i|V\rangle) \\
& \lambda_{2}=2, \quad|b\rangle=(1 / \sqrt{5})(2 i|H\rangle+|V\rangle) .
\end{aligned}
$$

Therefore, an interaction Hamiltonian of the form $H=g_{A} A P_{x}(B \equiv 0)$ describes a process where for an arbitrary input polarization, the $|a\rangle$ component is shifted by an amount $\Delta=g_{A} t$ while the $|b\rangle$ component is shifted by $2 \Delta$. The initial state of the system is $|i\rangle=|H\rangle$, and the initial shape of the pointer is characterized by the distribution $\phi(x, y)$ given by Eq. (3). The initial state of the system and pointer is factorable and is given by $\left|\psi^{\mathrm{in}}\right\rangle=$ $|H\rangle \otimes \int d x d y \phi(x, y)|x\rangle|y\rangle$.

The weak coupling interaction entangles the states of the system and pointer such that the resulting output state can be written as

$$
\begin{aligned}
\left|\psi^{\text {out }}\right\rangle= & \int d x d y[\phi(x-\Delta, y)|a\rangle \\
& -2 i \phi(x-2 \Delta, y)|b\rangle]|x, y\rangle .
\end{aligned}
$$

The output state is projected into a nearly orthogonal state for the system $|f\rangle=\sin \epsilon|H\rangle+\cos \epsilon|V\rangle$ so that $\langle f \mid i\rangle=\sin \epsilon$, with $\epsilon$ small. When considering the OAM pointer distribution given in Eq. (3), the expectation value of the pointer shift, up to second order in $\Delta$, is given by

$$
\langle X Y\rangle=-\frac{3 l}{5} \frac{\Delta^{2}}{\tan \epsilon} .
$$

Note that when using a Gaussian pointer state (here labeled with the subscript $\mathrm{G}$ ), we obtain $\langle X Y\rangle_{\mathrm{G}}=0$, and the usual one-dimensional mean value $\langle X\rangle_{\mathrm{G}}$ is

$$
\langle X\rangle_{\mathrm{G}}=\frac{9}{5} \Delta .
$$

We also compare the first order weak value, given by

$$
\langle A\rangle_{w}=\frac{9}{5}-i \frac{2}{5} \frac{1}{\tan \epsilon},
$$

with the higher order weak value, given by 


$$
\left\langle A^{2}\right\rangle_{w}=\frac{17}{5}-i \frac{6}{5} \frac{1}{\tan \epsilon} .
$$

Two points should be highlighted in this example. First, OAM pointer states allow retrieval of the imaginary part of the weak value $\left\langle A^{2}\right\rangle_{w}$, a feature which is not accessible with Gaussian pointer states. Second, Eq. (10) shows that there is no weak amplification in the position shift for a Gaussian pointer state. There is however amplification in the momentum shift $\left\langle P_{x}\right\rangle_{\mathrm{G}}$, which is related to the imaginary part of the weak value [Eq. (11)]. On the other hand, the higher order weak value [Eq. (12)] shows enhancement for small $\epsilon$. This, as shown in Eq. (5), allows for weak amplification of the two-dimensional position shift $\langle X Y\rangle$. We note that an important requirement in order to obtain such OAM enhancement is a nonvanishing imaginary part for the second order weak value (i.e., $\left.\operatorname{Im}\left(\left\langle A^{2}\right\rangle_{w}\right) \neq 0\right)$. This can, in turn, be interpreted as a specific form of ellipticity for the eigenvectors of the system observable $A$ [29].

To conclude, we presented a novel scheme for weak measurements that relies on the use of pointer states endowed with orbital angular momentum (OAM). We have shown that such higher-dimensional weak measurements can provide access to higher order weak moments of single-particle operators. In particular, for pointer states containing OAM with winding number $l= \pm 1$, it is possible to measure the weak value of the square of single-particle observables.

We have also shown that by considering a single-particle operator and a two-dimensional OAM pointer state, it is possible to measure an imaginary weak value via a twodimensional pointer position shift. This result is unexpected since it is usually believed that imaginary weak values are only accessible through the expectation value of the pointer's conjugate variable. In addition, we have demonstrated, by means of an example, that the use of OAM pointer states can provide weak amplification in configurations where the use of Gaussian pointer states cannot, thus allowing for a much wider range of applicability. For instance, one can engineer OAM states with higher winding numbers, or superpositions of OAM states, to obtain the sought-after higher order weak values that show the required enhancement.

The results presented here open the door to a number of novel fundamental and technological applications [16,21-23]. Furthermore, we emphasize that the use of pointer states with OAM is not restricted to radiation fields, and could also be envisioned in the context of Bose-Einstein condensates, where the coherent transfer of OAM of photons to matter can be used to create atomic vortex states [30].

This work was supported by the Government of Spain (Project FIS2010-14831) and project FET-Open 255914 (PHORBITECH). This work has also been supported by Fundacio Privada Cellex Barcelona. G. P. acknowledges financial support from Marie Curie International Incoming Fellowship COFUND. *graciana.puentes@icfo.es

[1] Y. Aharonov, D. Z. Albert, and L. Vaidman, Phys. Rev. Lett. 60, 1351 (1988).

[2] I. M. Duck, P. M. Stevenson, and E. C. G. Sudarshan, Phys. Rev. D 40, 2112 (1989).

[3] A. J. Leggett, Phys. Rev. Lett. 62, 2325 (1989); A. Peres, ibid. 62, 2326 (1989); Y. Aharonov and L. Vaidman, ibid. 62, 2327 (1989).

[4] Y. Aharonov and L. Vaidman, Phys. Rev. A 41, 11 (1990); J. M. Knight and L. Vaidman, Phys. Lett. A 143, 357 (1990).

[5] K. J. Resch and A. M. Steinberg, Phys. Rev. Lett. 92, 130402 (2004).

[6] G. T. Foster, L. A. Orozco, H. M. Castro-Beltran, and H. J. Carmichael, Phys. Rev. Lett. 85, 3149 (2000).

[7] H. M. Wiseman, Phys. Rev. A 65, 032111 (2002).

[8] D. R. Solli, C. F. McCormick, R. Y. Chiao, S. Popescu, and J. M. Hickmann, Phys. Rev. Lett. 92, 043601 (2004).

[9] N.S. Williams and A. N. Jordan, Phys. Rev. Lett. 100, 026804 (2008); M. E. Goggin, M. P. Almeida, M. Barbieri, B. P. Lanyon, J. L. O’Brien, A. G. White, and G. J. Pryde, Proc. Natl. Acad. Sci. U.S.A. 108, 1256 (2011); J. Dressel, C. J. Broadbent, J. C. Howell, and A. N. Jordan, Phys. Rev. Lett. 106, 040402 (2011).

[10] N. W. M. Ritchie, J. G. Story, and R. G. Hulet, Phys. Rev. Lett. 66, 1107 (1991).

[11] O. Hosten and P. Kwiat, Science 319, 787 (2008).

[12] N. Brunner and C. Simon, Phys. Rev. Lett. 105, 010405 (2010).

[13] P. B. Dixon, D. J. Starling, A. N. Jordan, and J. C. Howell, Phys. Rev. Lett. 102, 173601 (2009).

[14] J. S. Lundeen, B. Sutherland, A. Patel, C. Stewart, and C. Bamber, Nature (London) 474, 188 (2011).

[15] G. J. Pryde, J. L. O'Brien, A. G. White, T. C. Ralph, and H. M. Wiseman, Phys. Rev. Lett. 94, 220405 (2005).

[16] R. Raussendorf and H. J. Briegel, Phys. Rev. Lett. 86, 5188 (2001).

[17] Q. A. Turchette, C. J. Hood, W. Lange, H. Mabuchi, and H. J. Kimble, Phys. Rev. Lett. 75, 4710 (1995); J. D. Franson, Phys. Rev. Lett. 78, 3852 (1997).

[18] J. Dressel and A. N. Jordan, Phys. Rev. A 85, 012107 (2012).

[19] X. Zhu, Y. Zhang, S. Pang, C. Qiao, Q. Liu, and S. Wu, Phys. Rev. A 84, 052111 (2011); T. Koike and S. Tanaka, Phys. Rev. A 84, 062106 (2011); K. Nakamura, A. Nishizawa, and M. K. Fujimoto, Phys. Rev. A 85, 012113 (2012).

[20] Y. Susa, Y. Shikano, and A. Hosoya, Phys. Rev. A 85, 052110 (2012).

[21] Y. Hasegawa, R. Loidl, G. Badurek, M. Baron, and H. Rauch, Nature (London) 425, 45 (2003).

[22] M. Granata, C. Buy, R. Ward, and M. Barsuglia, Phys. Rev. Lett. 105, 231102 (2010).

[23] R. Brout, S. Massar, R. Parentani, and Ph. Spindel, Phys. Rep. 260, 329 (1995).

[24] A. Siegman, Lasers (University Science Books, Mill Valley, CA, 1986).

[25] L. Allen, M. W. Beijersbergen, R. J. C. Spreeuw, and J. P. Woerdman, Phys. Rev. A 45, 8185 (1992).

[26] J. P. Torres and L. Torner, Twisted Photons: Applications of Light with Orbital Angular Momentum (Wiley-Vch Verlag, Weinheim, 2011). 
[27] L. Allen, M. J. Padgett, and M. Babiker, Prog. Opt. 39, 291 (1999).

[28] M. A. Nielsen and I. L. Chuang, Quantum Computation and Quantum Information (Cambridge University Press, Cambridge, England, 2000).

[29] A particular example where our scheme would not retrieve any advantage over Gaussian pointer states is given for system observables satisfying $A^{2}=1$, such as the Pauli matrices. We note, however, that the maximum weak-value amplification factor that can be obtained in this particular case, employing Gaussian probe states, is also bounded [19].

[30] M. F. Andersen, C. Ryu, P. Clade, V. Natarajan, A. Vaziri, K. Helmerson, and W. D. Phillips, Phys. Rev. Lett. 97, 170406 (2006). 